\title{
SPECTRAL TYPE OF ONE-PARAMETER GROUP OF UNITARY OPERATORS WITH TRANSVERSAL GROUP
}

\author{
MASASI KOWADA
}

§ 1. Introduction. It is an important problem to determine the spectral type of automorphisms or flows on a probability measure space. We shall deal with a unitary operator $U$ and a 1-parameter group of unitary operators $\left\{U_{t}\right\}$ on a separable Hilbert space $H$, and discuss their spectral types, although $U$ and $\left\{U_{t}\right\}$ are not necessarily supposed to be derived from an automorphism or a flow respectively.

For our purpose, a transversal group, if it exists, plays an important role. A 1-parameter group $\left\{V_{s}\right\}$ of unitary operators is said to be a transversal group for $U$, if it satisfies

$$
U V_{s}=V_{\lambda s} U \quad \text { for some } \lambda \neq 0 .
$$

Similarly, $\left\{V_{s}\right\}$ is a transversal group for $\left\{U_{t}\right\}$, if it satisfies

$$
U_{t} V_{s}=V_{\text {sexp }(\lambda t)} U_{t} \quad \text { for some } \lambda \neq 0 \text {. }
$$

Ya. G. Sinai in [4] has already proposed the idea of a transversal field which is useful to see whether a given flow $\left\{T_{t}\right\}$ (an automorphism $A$ ) on a probability space $\Omega$ is Kolmogorov flow (Kolmogorov automorphism) or not.

For a flow $\left\{T_{t}\right\}$ (an automorphism $A$ ) on $(\Omega, P$ ), if there exists an another flow $\left\{Z_{s}\right\}$ on $(\Omega, P)$ such that

$$
T_{t} Z_{s}=Z_{u(s, t)} T_{t} \quad\left(A Z_{s}=Z_{u(s)} A\right),
$$

then the transversal field can be constructed as the measurable partition of the measure space $(\Omega, P)$ along the orbits of $\left\{Z_{s}\right\}$ in finite time intervals. Such a flow $\left\{Z_{s}\right\}$ induces a 1-parameter group of unitary operators $\left\{V_{s}\right\}$ on the space $L^{2}(\Omega)$ in a usual manner. The group $\left\{V_{s}\right\}$ satisfies the commutation relation mentioned above with $\left\{U_{t}\right\}$ induced by $\left\{T_{t}\right\}$ (with $U$

Received April 25, 1967. 
induced by $A$ ). Since we shall only deal with unitary operators, our discussions will start from the commutation relations with the transversal group. In our approach, although the structure of measure space never contributes, the existence of a transversal group gives us many informations about the spectral type of $U$ or $\left\{U_{t}\right\}$.

The main results in this paper read as follows

1) A unitary operator $U$ on a separable Hilbert space $H$ possesses Lebesgue spectrum, if there exists for $U$ a transversal group $\left\{V_{s}\right\}$ with $\lambda \neq 1$ (Theorem 3. 1).

2) A unitary operator $U$ possesses $\sigma$-Lebesgue spectrum, if $U$ has a transversal group $\left\{V_{s}\right\}$ with continuous spectrum (Theorem 3. 3).

3) A 1-parameter group of unitary operators $\left\{U_{t}\right\}$ on $H$ for which there exists a transversal group $\left\{V_{3}\right\}$ with $\lambda \neq 0$ has Lebesgue spectrum (Theorem 4. 3).

There are many important examples of unitary operators or 1-parameter groups of unitary operators with transversal groups such as flow of Brownian motion, geodesic flow on manifold of constant negative curvature and group automorphisms on $n$-dimensional torus. These examples can be investigated by our method as will be seen later.

The author would like to express his deep thanks to Professors T. Hida and $\mathrm{H}$. Nomoto for calling his attension to the paper [4] and for fruitful discussions which motivated this paper. Especially the author is greatly indebted to Professor $\mathrm{H}$. Nomoto for his help to improve the original form of this paper.

§ 2. The transversal group. We are informed rather poor knowledge about the property of 1-parameter group of unitary operators $\left\{U_{t}\right\}$ (a unitary operator $U$ ) if we regard $\left\{U_{t}\right\}(U)$ only to be a strongly continuous unitary representation of the additive group of real numbers (integers). We shall therefore introduce another 1-parameter group of unitary operators $\left\{V_{s}\right\}$ which transforms every orbit of $\left\{U_{t}\right\}(U)$ onto another.

Let $H$ be a separable Hilbert space.

Definition 2.1. A 1-parameter group of unitary operators $\left\{V_{s}\right\}$ on $H$ is a transversal group with $\lambda$ for a unitary operator $U$ on $H$ if they satisfy the commutation relation

$$
U V_{s}=V_{\lambda s} U,-\infty<s<+\infty
$$

for a nonzero real $\lambda$. 
Definition 2.2. A 1-parameter group of unitary operators $\left\{V_{s}\right\}$ on $H$ is a transversal group with $\lambda$ for a 1-parameter group $\left\{U_{t}\right\}$, if they satisfy the relation

$$
U_{t} V_{s}=V_{\operatorname{sexp}(\lambda t)} U_{t}, \quad-\infty<s, t<+\infty
$$

for a nonzero real $\lambda$.

The relations (2.1) and (2. 2) are the special cases of the followings;

$$
U V_{s}=V_{u(s)} U
$$

and

(2. 2$)^{\prime}$

$$
U_{t} V_{s}=V_{u(s, t)} U_{t}
$$

respectively.

Observing the relation that $V_{s}$ transforms $U_{t}$-orbits into themselves, (2. 1)' and $(2.2)^{\prime}$ seem to be more reasonable. However the following propositions 2.1 and 2.2 enable us to make use of (2.1) and (2.2) to define a transversal group.

Proposition 2. 1. Let $u=u(s)$ be a nonzero aperiodic measurable function. Then the relation

$$
U V_{s}=V_{u(s)} U
$$

implies that

$$
u(s)=\lambda s,
$$

for some $\lambda \neq 0$.

The proof follows from the assumptions and the following equalities

$$
U V_{(s+t)}=U V_{s} V_{t}=V_{u(s)} U V_{t}=V_{u(s)} V_{u(t)} U=V_{(u(s)+u(t))} U=V_{u(s+t)} U \text {. }
$$

Proposition 2.2. Suppose that $V_{s}$ is aperiodic and $u=u(t, s\rangle$ is a nonzero measurable function with respect to both $t$ and $s$. Then the relation

$$
U_{t} V_{s}=V_{u(t, s)} U_{t}
$$

implies that

$$
u(t, s)=\mu_{s} \exp (\lambda t)
$$

for some nonzero $\mu$ and $\lambda$. 
Proof. From the similar calculation to the previous proposition, we see that $u(t, s)=s v(t)$, for some function $v(t)$. We have

$$
\begin{aligned}
U_{t_{1}+t_{2}} V_{s} & =U_{t_{1}} V_{u\left(t_{2}, s\right)} U_{t_{2}}=V_{u\left(t_{1}, u\left(t_{2}, s\right)\right)} U_{t_{1}+t_{2}} \\
& =V_{u\left(t_{1}+t_{2}, s\right)} U_{t_{1}+t_{2}}
\end{aligned}
$$

and concequently

$$
\begin{aligned}
u\left(t_{1}+t_{2}, s\right) & =s v\left(t_{1}+t_{2}\right) \\
& =u\left(t_{1}, u\left(t_{2}, s\right)\right)=u\left(t_{2}, s\right) v\left(t_{1}\right)=s v\left(t_{1}\right) v\left(t_{2}\right) .
\end{aligned}
$$

It follows that $v(t)=\mu \exp (\lambda t)$ for some $\mu \neq 0$ and $\lambda \neq 0$. This completes the proof.

Throughout this paper, we assume that

$$
H \text { is a separable Hilbert space, }
$$

and

$$
N \equiv\left\{\phi \in H: V_{s} \phi=\phi \text { for all } s\right\}=\{0\} .
$$

In the case where $(B)$ is not fulfilled, the simultaneous reduction of $\left\{U_{t}\right\}(U)$ and $\left\{V_{s}\right\}$ onto $H \ominus N$ leads to our situation.

\& 3. Spectral type of a unitary operator with transversal group. In this section, we assume that $U$ has a transversal group $\left\{V_{s}\right\}$ with $|\lambda| \neq 1$. The proof will be given only in the case where $|\lambda|<1$. In case $U$ has a transversal group $\left\{V_{s}\right\}$ with $|\lambda|>1$, similar discussions lead us to the same results.

Let $\Gamma$ be the set of all proper values of $\left\{V_{s}\right\}$, and for some positive $\mu_{0}$, put $I_{m}^{+}=\left[\mu_{0} /|\lambda|^{m}, \mu_{0} /|\lambda|^{m+1}\right]$ and $I_{m}^{-}=-I_{m}^{+}(m=0, \pm 1, \pm 2, \cdots)$. Then we get a partition of $\Gamma$

$$
\Gamma=\bigcup_{m=-\infty}^{+\infty}\left\{\Gamma_{m}^{+} \cup \Gamma_{m}^{-}\right\}
$$

where

$$
\begin{aligned}
& \Gamma_{m}^{+}=\Gamma \cap I_{m}^{+} \\
& \Gamma_{m}^{-}=\Gamma \cap I_{m}^{-}
\end{aligned}
$$

Lemma 3.1. We have

$$
\begin{array}{ll}
\Gamma_{m}^{ \pm}=\lambda^{-m} \Gamma_{0}^{ \pm}, & \text {if } \lambda>0, \text { or if } \lambda<0 \text { and } m \text { is even, } \\
\Gamma_{m}^{ \pm}=\lambda^{-m} \Gamma_{0}^{\ddagger}, & \text { if } \lambda<0 \text { and } m \text { is odd. }
\end{array}
$$


Proof. Suppose $V_{s} \varphi=\exp (i s \mu) \varphi$ for any $s$. Then, from (2.1), we get

$$
V_{s} U^{m} \varphi=U^{m} V_{s \lambda^{-m}} \varphi=\exp \left(i s \mu \lambda^{-m}\right) U^{m} \varphi,
$$

that is, $\mu \lambda^{-m} \in \Gamma(m=0, \pm 1, \pm 2, \cdots)$, and it is easy to get the conclusion.

Denote the proper vectors by $\varphi_{n}^{k}$ corresponding to $\mu_{k} \in \Gamma_{0}^{+} \cup \Gamma_{0}^{-}(k=$ $1,2, \cdots, n=1,2, \cdots, n_{k}$, where $n_{k}$ is the multiplicity of $\left.\mu_{k}\right)$. Then, for all $s$,

$$
\begin{aligned}
& \left(U^{m} \varphi_{n}^{k}, U^{m \prime} \varphi_{n^{\prime}}^{k^{\prime}}\right)=\left(V_{s} U^{m} \varphi_{u}^{k}, V_{s} U^{m^{\prime}} \varphi_{k^{\prime}}^{k^{\prime}}\right) \\
& \left.=\left(U^{m} V_{s \lambda^{-m}} \varphi_{t s}^{k}, U^{m} V_{s \lambda^{-m^{\prime}}} \varphi_{k^{\prime}}^{k^{\prime}}\right)\right) \\
& =\exp \left[i s\left(\mu_{k} \lambda^{-m}-\mu_{k^{\prime}} \lambda^{-m^{\prime}}\right)\right]\left(U^{m} \varphi_{l^{\prime}}^{k}, U^{m^{\prime}} \varphi_{n^{\prime}}^{k^{\prime}}\right)
\end{aligned}
$$

and

$$
\left(U^{m} \varphi_{l i}^{k}, U^{m} \varphi_{b^{\prime}}^{k}\right)=\left(\varphi_{l, k}^{k}, \varphi_{u^{\prime}}^{k}\right)
$$

These equalities imply

$$
U^{m} \varphi_{n}^{k} \perp U^{m \prime} \varphi_{n^{\prime}}^{k^{\prime}},
$$

unless $\mu_{k}=\mu_{k^{\prime}}, m=m^{\prime}$ and $n=n^{\prime}$ hold simultaneously.

Consequently, denoting $H_{n}^{k}=\subseteq\left\{U^{m} \varphi_{n}^{k}: m=0, \pm 1, \cdots\right\}$, we have

$$
H_{n}^{k} \perp H_{n^{\prime}}^{k^{\prime}} \quad\left(k \neq k^{\prime} \text { or } n \neq n^{\prime}\right)
$$

Lemma 3.2. The operator $U$ has simple Lebesgue spectrum on each cyclic subspace $H_{n}^{k}$.

Proof. Denoting

$$
U^{m}=\int e^{i m \hat{\xi}} d E^{U}(\xi),
$$

and noticing (3.2), we have

$$
\int e^{i m \xi} d\left\|E^{U}(\xi) \varphi_{n}^{k}\right\|^{2}=\left(U^{m} \varphi_{n}^{k}, \varphi_{l i}^{k}\right)=0
$$

for any $n, k$ and $m \neq 0$. Paley-Wiener's theorem, therefore, implies the conclusion.

THEOREM 3.1. Suppose that for a unitary operator $U$ on a separable Hilbert space $H$, there exists a transversal group $\left\{V_{s}\right\}$ with $|\lambda| \neq 1$ which has purely point spectrum. Then $U$ has $\left(\sum n_{k}\right)$-multiple Lebesgue spectrum. 
Proof. Denote by $M_{m}^{+}\left(M_{m}^{-}\right)$the subspace of $H$ spanned by all proper vectors corresponding to $\Gamma_{m}^{+}\left(\Gamma_{m}^{-}\right)$. Then, from (3. 1), we get

$$
H=\Sigma \oplus\left(M_{m}^{+} \oplus M_{m}^{-}\right) .
$$

While the equality $U^{m} M_{n}^{ \pm}=M_{n+m}^{ \pm}\left(U^{m} M_{n}^{ \pm}=M_{n+m}^{\mp}\right)$, and the construction of $H_{u}^{k}$ shows that

$$
U^{m} M_{0}^{+}\left(U^{m} M_{0}^{-}\right) \subset \Sigma \oplus H_{n}^{k} \subset H
$$

for $m=0, \pm 1, \cdots$. This leads us to the conclusion

$$
\sum_{n, k} \oplus H_{n}^{k}=H .
$$

Hence, combining (3.6) with the lemma 3.2, we see that the spectral measure of $U$ does not have singular component with respect to Lebesgue measure, and that $H_{n}^{k}$ appears in the direct sum as many times as $\sum n_{k}$. This completes the proof.

ExAmple 3.1. A group automorphism on the torus. Let $T^{n}$ be the $n$-dimensional torus and $A$ be a group automorphism on $T^{n}$. It is known that $A$ can be written in a matrix form. Suppose that $A$, regarded as a transformation on $n$-dimensional vector space $R^{n}$, has a real proper value $\lambda$ such that $|\lambda| \neq 1$ and has a proper vector $r$ corresponding to $\lambda$. Let $\left\{g_{s}\right\}$ be a 1-parameter subgroup of $T^{n}$ defined by

$$
\left(g_{s}, g^{\wedge}\right)=\exp i s<r, g^{\wedge}>, g^{\wedge} \in\left(T^{n}\right)^{\wedge},
$$

where $\left(T^{n}\right)^{\wedge}$ is the dual group of $T^{n}$ and $\left\langle r, g^{\wedge}\right\rangle$ is the inner product in $R^{n}$. Then $A$ and the flow $\left\{Z_{s}\right\}$ which is defined by

$$
Z_{s} g=g+g_{s}, \quad g \in T^{n}
$$

induce a unitary operator $U$ and 1-parameter group of unitary operators $\left\{V_{s}\right\}$ on $L^{2}\left(T^{n}\right)$ in a usual manner. It can be easily seen that $\left\{V_{s}\right\}$ is a transversal group for $U$ and that $\left\{V_{s}\right\}$ has the purely point spectrum. Consequently, according to the theorem 3.1, $U$ has uniform Lebesgue spectrum. Noticing that a unitary operator which has a uniform Lebesgue spectrum is ergodic, we can show that the unitary operator $U$ has .exactly $\sigma$-Lebesgue spectrum.

Next we shall deal with a unitary operator $U$ which has a transversal group $\left\{V_{s}\right\}$ with continuous spectrum. Let 


$$
V_{s}=\int_{-\infty}^{+\infty} e^{2 \pi i s \xi} d E^{V}(\xi)
$$

and

$$
H_{\xi}=E^{V}(\xi) H
$$

Theorem 3. 2. For any $\xi$ and $m=0$, $\pm 1, \pm 2, \cdots$, we get

$$
U^{m} H_{\hat{\xi}}= \begin{cases}H_{\xi \lambda^{-m}} & \text { if } \lambda>0, \text { or } \lambda<0 \text { and } m \text { is even } \\ H_{\xi \lambda-m}^{c} & \text { if } \lambda<0 \text { and } m \text { is odd, }\end{cases}
$$

where $H_{\xi}^{c}$ means the orthogonal complement of $H_{\xi}$.

Proof. Suppose that $\lambda>0$, or $\lambda<0$ and $m$ is even. Then we have

$$
\begin{aligned}
\left(V_{s} f, g\right) & =\int_{-\infty}^{+\infty} e^{2 \pi i s \xi} d\left(E^{V}(\xi) f, g\right) \\
& =\left(U^{m} V_{s} f, U^{m} g\right) \\
& =\left(V_{\lambda^{m}} U^{m} f, U^{m} g\right) \\
& =\int_{-\infty}^{+\infty} e^{2 \pi i s \hat{\xi}} d\left(E^{V}\left(\xi \lambda^{-m}\right) U^{m} f, U^{m} g\right) .
\end{aligned}
$$

Clearly $\left\{U^{-m} E^{v}\left(\xi \lambda^{-m}\right) U^{m},-\infty<\xi<+\infty\right\}$ is a resolution of the identity and hence the uniqueness of a resolution implies

$$
U^{m} E^{V}(\xi)=E^{V}\left(\xi \lambda^{-m}\right) U^{m}
$$

Consequently we get

$$
U^{m} H_{\xi}=H_{\xi \lambda^{-m}} \text {. }
$$

Next we suppose that $\lambda<0$ and $m$ is odd. Put $F(\eta)=I-E^{V}\left(\lambda^{-m} \eta-\right)$, where $I$ is the identity operator. Clearly $\{F(\eta)\}$ is the resolution of the identity. We get

$$
\begin{aligned}
(V, f, g) & =\int_{-\infty}^{+\infty} e^{2 \pi i s \xi \lambda^{m}} d\left(E^{V}(\xi) U^{m} f, U^{m} g\right) \\
& =\int_{-\infty}^{+\infty} e^{2 \pi i s \xi \lambda^{m}} d\left(\left(I-F\left(\lambda^{m} \xi\right)\right) U^{m} f, U^{m} g\right) \\
& =\int_{+\infty}^{-\infty} e^{2 \pi i s \eta} d\left((I-F(\eta)) U^{m} f, U^{m} g\right) \\
& =\int_{-\infty}^{+\infty} e^{2 \pi i s \eta} d\left(F(\eta) U^{m} f, U^{m} g\right) .
\end{aligned}
$$

From these we get $U^{m}-E^{v}\left(\lambda^{-m} \eta\right) U^{m}=U^{m} E^{v}(\eta)$ and hence 


$$
U^{m} H_{\xi}=H_{\xi \lambda-m}^{c}
$$

This completes the proof.

Put $M_{m}^{ \pm}=E^{V}\left(I_{m}^{ \pm}\right) H$. We can easily see that $\left\{U^{m} M_{n}^{ \pm}: m, n=0, \pm 1, \cdots\right\}$ is an orthogonal family of subspaces, and moreover, from (3. 9), we have

$$
U^{m} M_{b b}^{ \pm}= \begin{cases}M_{n+m}^{ \pm} & \text {if } \lambda>0, \text { or if } \lambda<0 \text { and } m \text { is even } \\ M_{n+m}^{\mp} & \text { if } \lambda<0 \text { and } m \text { is odd. }\end{cases}
$$

Let $\left\{\varphi_{k}^{+}\right\}\left(\left\{\varphi_{k}^{-}\right\}\right)$be a basis of $M_{0}^{+}\left(M_{0}^{-}\right)$and let $H_{k}^{+}\left(H_{k}^{-}\right)$be the cyclic subspace spanned by $\left\{U^{m} \varphi_{k}^{+}: m=0, \pm 1, \cdots\right\}\left(\left\{U^{m} \varphi_{k}^{-}: m=0,1, \cdots\right\}\right)$. Then the equalities (3.11) imply that for $k=1,2, \cdots$

$$
\int e^{2 \pi i m \xi} d\left\|E^{U}(\xi) \varphi_{k}^{ \pm}\right\|^{2}=\left(U^{m} \varphi_{k}^{ \pm}, \varphi_{k}^{ \pm}\right)=0 \quad(m \neq 0)
$$

Paley-Wiener's theorem, therefore, implies that $U$ has simple Lebesgue spectrum on each cyclic subspace $H_{k}^{ \pm}$.

Clearly

$$
\begin{aligned}
H & =\Sigma \oplus\left(M_{m}^{+} \oplus M_{m}^{-}\right)=\Sigma \oplus\left(U^{m} M_{0}^{+} \oplus U^{m} M_{0}^{-}\right) \\
& =\Sigma \oplus\left(H_{k}^{+} \oplus H_{k}^{-}\right) .
\end{aligned}
$$

The subspaces $M_{0}^{+}$and $M_{0}^{-}$are invariant under the group $\left\{V_{s}\right\}$, and $\left\{V_{s}\right\}$ does not have proper value in $M_{0}^{+}$and $M_{0}^{-}$. Consequently the dimension of $M_{0}^{+}\left(M_{0}^{-}\right)$must be infinite. This means that the components $H_{k}^{+}\left(H_{k}^{-}\right)$ appear infinitely many times in the decomposition (3. 12).

Summing up the above discussion we get

Theorem 3. 3. A unitary operator $U$ on a separable Hilbert space $H$ has $\sigma$-Lebesgue spectrum, if there exists a transversal group $\left\{V_{s}\right\}$ on $H$ with $|\lambda| \neq 1$ the spectrum of which is continuous.

For a unitary operator $U$, if there exists a transversal group $\left\{V_{s}\right\}$ with $|\lambda| \neq 1$ whose spectrum is not necessarily purely discrete nor purely continuous, we may reduce $U$ and $H$ to those appeared in the previous two particular cases. Let us denote by $H_{d}$ and $H_{c}$ the subspaces spanned by all of the proper vectors of $\left\{V_{s}\right\}$ and its orthogonal subspace, respectively. We shall now discuss the case where both $H_{d}$ and $H_{c}$ are nontrivial.

TheOREM 3. 4. If a unitary operator $U$ on a separable Hilbert space $H$ has 
a transversal group $\left\{V_{s}\right\}$ with $|\lambda| \neq 1$ on $H$, and if both $H_{d}$ and $H_{c}$ are nontrivial, then $U$ has $\sigma$-Lebesgue spectrum.

Proof. Let $P_{d}$ be the projection operator on $H_{d}$, and put $P_{d} V_{s}=V_{s}^{d}$ and $P_{d} U=U^{d}$. Clearly $P_{d}$ commutes with $V_{s}(-\infty<s<+\infty)$. From Lemma 3. 1, we get, for any $f \in H_{d}, U f \in H_{d}$ and $U^{*} f \in H_{d}$. Thus $U$ reduces the subspace $H_{d}$. This means that $U$ commutes with $P_{d}$, so we have

$$
\begin{aligned}
U^{d} V_{s}^{d} & =P_{d} U P_{d} V_{s}=P_{d} U V_{s}=P_{d}^{2} V_{\lambda s} U \\
& =P_{d} V_{\lambda s} P_{d} U=V_{\lambda_{s}}^{d} U^{d} .
\end{aligned}
$$

Thus $U^{d}$ has a transversal group $\left\{V_{s}^{d}\right\}$ with the same $\lambda$.

Put $\left(I-P_{d}\right) U=U^{c}$ and $\left(I-P_{d}\right) V_{s}=V_{s}^{c}$. Then $\left\{V_{s}^{c}\right\}$, likewise the above (3. 20) constitutes the transversal group of $U^{c}$, and according to Theorem 3. 1 and 3. 3, we see that $U$ has Lebesgue spectrum on $H=H_{d}+H_{c}$, and moreover, at least on $H_{c}, U$ has $\sigma$-Lebesgue spectrum. This completes the proof.

§4. Spectral type of a 1-parameter group of unitary operators with a transversal group. Even in case of a 1-parameter group of unitary operators, the analogous discussions to the previous section goes on with a few modifications.

A typical difference between the case of a single unitary operator and that of a 1-parameter group of unitary operators is the fact that only the latter has no transversal group whose spectral measure contains discrete component except point measure concentrating at 0 .

Theorem 4. 1. The transversal group $\left\{V_{s}\right\}$ of $\left\{U_{t}\right\}$ has purely continuous spectrum on a separable Hilbert space $H$.

Proof. Suppose there exists $\mu \neq 0$ such that

$$
V_{s} \varphi=e^{i s \mu} \varphi
$$

for all s. Then we get

$$
\begin{aligned}
\left(U_{t_{1}} \varphi, U_{t_{2}} \varphi\right) & =\left(V_{s} U_{t_{1}} \varphi, V_{s} U_{t_{2}} \varphi\right) \\
& =\exp \left(i s \mu e^{\lambda\left(t_{1}-t_{2}\right)}\right) \quad\left(U_{t_{1}} \varphi, U_{t_{2}} \varphi\right) .
\end{aligned}
$$

This implies that $U_{t_{1}} \varphi$ is orthogonal to $U_{t_{2}} \varphi$, if $t_{1} \neq t_{2}$. In other words, 
$\left\{U_{t} \varphi:-\infty<t<+\infty\right\}$ is an orthogonal system in $H$. This contradicts the separability of $H$.

For convenience, we shall discuss a group $\left\{U_{t}\right\}$ which has a transversal group $\left\{V_{s}\right\}$ with negative $\lambda$. Because, if $\lambda>0$, we can proceed to the same conclusion with slight modification.

We shall omit the proof of the following proposition which is similar to the proof of (3.9).

Theorem 4. 2. For any $\xi$ and $t$, we get

$$
U_{t} H_{\xi}=H_{\xi} e^{-\lambda t} \text {. }
$$

Put $M(t, \xi)=H_{\xi} e^{-\lambda t} \ominus H_{\xi}$. Then, for suitable $t=t_{0}$ and $\xi=\xi_{0}>0$, $M^{+}=M\left(t_{0}, \xi_{0}\right)$ is an infinite dimensional subspace, because $e^{-\lambda t}$ is strictly increasing. Let $\left\{\varphi_{n}\right\}$ be a basis of $M^{+}$. If $|t|>t_{0}$,

$$
U_{t} \varphi_{1} \in U_{t} H_{\xi_{0} e^{-\lambda} t_{0}} \ominus U_{t} H_{\xi_{0}} \subset H_{\left.\xi_{0} e^{-\lambda\left(t+t_{0}\right.}\right)} \ominus H_{\xi_{0} e^{-\lambda} t_{0}} .
$$

Consequently we get, if $|t| \geq t_{0}$,

$$
\left(U_{t} \varphi_{1}, \varphi_{1}\right)=0 .
$$

This shows us that $\left\{U_{t}\right\}$ has simple Lebesgue spectrum on the cyclic subspace $H_{1}^{+}$spanned by $\left\{U_{t} \varphi_{1},-\infty<t<+\infty\right\}$. Let $P_{1}$ be the projection operator on $H_{1}^{+}$. If $\varphi_{2}=P_{1} \varphi_{2}$, we proceed to $\varphi_{3}$. If $\varphi_{2} \neq P_{1} \varphi_{2}$, we shall denote by $H_{2}^{+}$the cyclic subspace spanned by $\left\{U_{t}\left(\varphi_{2}-P_{1} \varphi_{2}\right),-\infty<t<+\infty\right\}$. From the equalities

$$
\begin{aligned}
& \left(U_{t}\left(\varphi_{2}-P_{1} \varphi_{2}\right), \quad\left(\varphi_{2}-P_{1} \varphi_{2}\right)\right) \\
& \quad=\left(U_{t} \varphi_{2}, \varphi_{2}\right)-\left(U_{t} P_{1} \varphi_{2}, P_{1} \varphi_{2}\right) \\
& \quad=-\left(U_{t} P_{1} \varphi_{2}, P_{1} \varphi_{2}\right), \quad|t|>t_{0},
\end{aligned}
$$

we see that $\left\{U_{t}\right\}$ also has simple Lebesgue spectrum on $H_{2}^{+}$. Continuing such a procedure, we obtain a sequence of cyclic subspaces, $H_{1}^{+}, H_{2}^{+}, \cdots$, on each of which $\left\{U_{t}\right\}$ has simple Lebesgue spectrum. Noticing that $\varphi_{n} \in H_{1}^{+} \oplus \cdots \oplus H_{n}^{+}$for any $n$, we get

$$
M^{+} \subset \Sigma \oplus H_{u}^{+} \subset H \text {. }
$$

Starting from $M^{-}=M\left(t_{1}, \xi_{1}\right)$ where $t_{1}$ and $\xi_{1}$ are suitable negative real numbers, the similar construction of the cyclic subspaces $H_{n}^{-}(n=1,2, \cdots)$ yields 


$$
M^{-} \subset \Sigma \oplus H_{n}^{-} \subset H \text {. }
$$

Evidently, $\Sigma \oplus H_{n}^{+}$is orthogonal to $\Sigma \oplus H_{n}^{+}$. We finally show that $H$ can be expressed as the direct sum of them.

We obtain

$$
\begin{aligned}
& \left(U_{t} H_{\xi_{0}}\right) \ominus H_{0}=U_{t-t_{0}}\left(H_{\xi_{0}} e^{-\lambda t_{0}} \ominus H_{\xi_{0}}\right) \oplus U_{t-2 t_{0}}\left(H_{\xi_{0}} e^{-\lambda t} \Theta_{0} \ominus H_{\xi_{0}}\right) \oplus \cdots \\
& =U_{t-t_{0}} M^{+} \oplus U_{t-2 t_{0}} M^{+} \oplus \cdots \cdot \text {, } \\
& U_{t} H_{\xi_{1}}=U_{t}\left(H_{\xi_{1}} e^{-\lambda t_{1}} \ominus H_{\xi_{1}}\right) \oplus U_{t+t_{1}}\left(H_{\xi_{1}} e^{-\lambda t_{1}} \ominus H_{\xi_{1}}\right) \oplus \\
& =U_{t} M^{-} \oplus U_{t+t_{1}} M^{-} \oplus \cdots \cdot
\end{aligned}
$$

These imply

$$
U_{t} H_{\xi_{0}} \subset\left(\Sigma \oplus H_{n}^{+}\right) \oplus H_{0}
$$

and

$$
U_{t} H_{\xi_{1}} \subset \Sigma \oplus H_{n}^{-} \subset H_{0} .
$$

While the continuity of $E^{V}(\xi)$ and the equation

$$
U_{t} H_{-\xi_{0}}=H_{-\xi_{0} e^{-\lambda t}}=E^{V}\left(-\xi_{0} e^{-\lambda t}\right) H
$$

imply

$$
H_{0}=E^{V}(0) H=\Sigma \oplus H_{n}^{-} .
$$

Consequently we have

$$
U_{t} H_{\xi_{0}} \subset \Sigma \oplus\left(H_{n}^{+} \oplus H_{n}^{-}\right) .
$$

Now the concluding result

$$
\Sigma \oplus\left(H_{n}^{+} \oplus H_{n}^{-}\right)=H
$$

can be deduced from the followings

$$
\begin{aligned}
& U_{t} H_{\hat{\xi}_{0}}=H_{\xi_{0} e^{-\lambda t}}=E^{V}\left(\xi_{0} e^{-\lambda t}\right) H \\
& E^{V}\left(\xi_{0} e^{-\lambda t}\right) \longrightarrow I \quad \text { (the eidentity operator) as } t \longrightarrow+\infty .
\end{aligned}
$$

Remark that, contrasting to $\S 3$, we have no information about the number of components in the direct sum $\Sigma \oplus\left(H_{n}^{+} \oplus H_{n}^{-}\right)$.

Summing up, we get

TheOREM 4.3. A 1-parameter group of unitary operators $\left\{U_{t}\right\}$ on a separable Hilbert space $H$ has uniform Lebesgue spectrum, if there exists a transversal group $\left\{V_{s}\right\}$ with $\lambda \neq 0$ for $\left\{U_{t}\right\}$. 
Example 4.1. A geodesic flow on a manifold of constant negative curvature. Let us denote the Lie group $S L(2, R)$ by $G$ and denote by $D$ some discrete subgroup of $G$ such that the measure $\mu$ on the homogenuous space $M=G / D$ induced from a left invariant measure on $G$ is finite. Then a 1-parameter subgroup $\left\{g_{t}\right\}$ of $G$ induces a group of left transformations on $M$ defined by

$$
g D \in M \longrightarrow g_{t} g D \in M .
$$

The dynamical system $\left(M, \mu,\left\{g_{t}\right\}\right)$ has a realization as a geodesic flow on a manifold of constant negative curvature (refer to [1]).

Let $g$ be the generator of $\left\{g_{t}\right\}$ and $h$ be a solution of the equation

$$
[g, h]=\lambda h
$$

for some $\lambda \neq 0$. Put $h_{s}=e^{s h}$. Then it is straightforward to see that the relation (2.2) is satisfied by 1-parameter groups of unitary operators $\left\{U_{t}\right\}$ and $\left\{V_{s}\right\}$ which are induced in a usual manner on $L^{2}(M)$ by $\left\{g_{t}\right\}$ and $\left\{h_{s}\right\}$ respectively. Concequently, from Theorem 4. 3, we see that $\left\{U_{t}\right\}$ has uniform Lebesgue spectrum. In [1] Gelfand and Fomin have shown that $\left\{U_{t}\right\}$ has exactly $\sigma$-Lebesgue spectrum, appealing to the automorphic function theory.

Example 4.2. A flow of the generalized white noise ${ }^{(*)}$. Let $S$ be Schwartz's space and $S^{*}$ be its conjugate space. Consider the probability space $P=\left(S^{*}, B, \mu\right)$, where $B$ is the Borel field generated by cylindrical sets in $S^{*}$ and $\mu$ is a white noise. The characteristic functional of $\mu$ is given by

$$
C_{\alpha}(\xi)=\exp \left\{-\frac{1}{\alpha} \int|\xi(t)|^{\alpha} d t\right\} .
$$

Let $\tau_{s}$ be the shift operator in $S$ defined by

$$
\tau_{s} \xi(t)=\xi(t-s), \quad \xi \in S,
$$

and we shall introduce the transformation $T_{t}$ on $S$ defined by

$$
T_{t} \xi(s)=e^{\frac{\lambda t}{\alpha}} \xi\left(e^{\lambda t} s\right),
$$

(*) The reader is referred to the forthcoming paper of $\mathrm{H}$. Nomoto and T. Hida for more detailed discussions. The above example is due to them (oral communication). 
where $\lambda$ is a nonzero real. Then, on the Hilbert space $H$ with the reproducing kernel $C_{\alpha}(\xi-\eta)$, we get 1-parameter groups of unitary operators $\left\{U_{t}\right\}$ and $\left\{V_{s}\right\}$ induced by $U_{t} f(\xi)=f\left(T_{t} \xi\right)$ and $V_{s} f(\xi)=f\left(\tau_{s} \xi\right)$, respectively. They satisfy the relation (2. 2), and hence, from the Theorem 4.3 , we see that $\left\{U_{t}\right\}$ has uniform Lebesgue spectrum.

\section{REFERENCES}

[1] I.M. Gelfand and S.V. Fomin: A geodesic flow on manifold of constant negative curvature, Uspehi Math. Nauk, t. VII, b. I (1952), 118-137.

[2] P.R. Halmos: Lectures on ergodic theory, 1956.

[ 3 ] J. von Neumann: Zur Operatorenmethode in der klassischen Mechanik, Ann. of Math., 33 (1932), 587-642.

[4] Ya. G. Sinai: Dynamical systems with countable Lebesgue spectrum II, Izv. AN. SSSR, 30 (1966), 15-68. (in Russian)

[5] H. Totoki: Flow and Entropy, Seminar on Prob., Vol. 20 (1964) (in Japanese)

Nagoya Institute of Technology 